\title{
Measuring density-driven errors using Kohn-Sham inversion
}

\author{
Seungsoo Nam, Suhwan Song, And Eunji Sim* \\ Department of Chemistry, Yonsei University, 50 Yonsei-ro Seodaemun-gu, Seoul 03722, Korea \\ KiERON BURKE \\ Departments of Chemistry and of Physics, University of California, Irvine, CA 92697, USA
}

April 27, 2020

\begin{abstract}
Kohn-Sham inversion, that is, the finding of the exact Kohn-Sham potential for a given density, is difficult in localized basis sets. We study the precision and reliability of several inversion schemes, finding estimates of density-driven errors at a useful level of accuracy. In typical cases of substantial density-driven errors, HF-DFT is almost as accurate as DFT evaluated on $\operatorname{CSSD}(T)$ densities. A simple approximation in practical HF-DFT also makes errors much smaller than the density-driven errors being calculated. Two paradigm examples, stretched $\mathrm{NaCl}$ and the $\mathrm{HO}^{-\mathrm{Cl}^{-}}$radical, illustrate just how accurate $H F-D F T$ is.
\end{abstract}

\section{INTRODUCTION}

Kohn-Sham (KS) density functional theory (DFT)[1] is an extremely popular approach to electronic structure problems, but the quality of the results depends on the quality of the exchange-correlation (XC) approximation used. Because the $\mathrm{KS}$ equations are solved self-consistently, there are errors in both the self-consistent (SC-) energy and the SC-density. [2] In most KS calculations, the density errors contribute little to the overall energy error. However, in various generic situations, semilocal approximations to XC make unusually large density errors (called density-driven errors) and the density error contributes significantly to the resulting energy error. In modern DFT parlance, these are attributed to delocalization errors of the density. [3]

The theory behind density-corrected (DC) DFT explains the origin of such errors, when they are likely to be significant, and how they can usually be reduced by using a more accurate density. [2, 4, 5] The exact density-driven error is defined as the difference in energy when the approximate functional is evaluated on its $\mathrm{SC}$ and exact densities. If the exact density was needed to perform a DC-DFT calculation, the procedure would be impractical, as finding a highly accurate density is more costly than the DFT calculation itself. However, in practice, for many semilocal approximations applied to molecular properties, it has been found that most density-driven errors can be greatly reduced by use of the Hartree-Fock (HF) density instead of the exact density. As the HF density is of comparable cost to DFT, this leads to a very practical approach (HF-DFT) which can be implemented very rapidly and costs no more than a typical DFT calculation. [6] HF-DFT has been used to reduce density-driven errors for electron affinities, [7. potential energy curves, [8, 9] spin gaps for coordination compound, [10] and noncovalent interactions. [11]

Given these successes of HF-DFT, we now ask: Can its

\footnotetext{
*esim@yonsei.ac.kr
}

underlying assumptions be tested? The most important assumption is that, when density-driven errors are significant, in molecules, the HF density yields more accurate energies than the $\mathrm{SC}$ density. A lesser assumption is that, in practical HFDFT calculations, the difference between HF and KS kinetic energies are ignored. The answer is yes, by employing the well-established technique of KS inversion to highly accurate densities, in order to extract exact density-driven errors and compare with the HF-DFT procedure. KS inversion is the process of finding an accurate KS potential (and associated objects, such as KS kinetic energy, HOMO, etc.) from a given density. From very early on, 12] method-developers in DFT have sought such exact information. [13, 14, 15, 16, 17] But most such inversions have been focussed on specific quantities such as eigenvalues, which can be very sensitive to the details of the density.

Here, we apply standard KS inversion procedures with the sole focus of testing the assumptions underlying HF-DFT. We use inversions to calculate density-driven errors for typical systems in which HF-DFT has proven successful. With two standard methods, we explore both the dependence on the basis set and the guiding density functional used (defined later). However, there are well-documented difficulties[18, 19 [20, 21] when such inversions are performed in finite localized basis sets. We find that methods to overcome such difficulties, while imprecise, yield sufficient accuracy to answer the most basic questions about the density-driven error. These methods, applied to HF and high-level ab initio densities in standard basis sets, produce sufficiently accurate density-driven error estimates to usefully address such questions, i.e., their remaining errors are small relative to common density-driven errors. We also find that the approximation used in practical HF-DFT calculations, namely using the HF kinetic energy instead of the KS kinetic energy, typically leads to changes of $1-2 \mathrm{kcal} / \mathrm{mol}$, which is below a standard threshold for declaring a density-driven error. [22] 
The paper is organized as follows. In Section II, we present backgrounds about wavefunctions and KS-DFT, KS inversion, and DC-DFT. Section III shows inversion results and gives some discussions about the uncertainty of the inversion, together with testing DC-DFT. Finally, we deduce our conclusion from the discussions.

\section{BACKGROUND}

\section{i. Wavefunctions and Kohn-Sham DFT}

Start from the variational principle for the exact ground-state energy

$$
E_{v}=\min _{\Psi}\langle\Psi|\hat{H}| \Psi\rangle,
$$

where $\hat{H}$ is the $N$-electron Hamiltonian with one-body potential $v(\mathbf{r})$, and the search is over all antisymmetric, normalized many-body wavefunctions $\Psi$. A HF calculation uses only a single Slater determinant, denoted $\Phi$ (assuming for now no symmetry breaking):

$$
E_{v}^{\mathrm{HF}}=\min _{\Phi}\langle\Phi|\hat{H}| \Phi\rangle
$$

where $\Phi_{v}^{\mathrm{HF}}$ denotes the minimizer. The traditional definition of the correlation energy is then

$$
E_{\mathrm{C}, v}^{\mathrm{trad}}=E_{v}-E_{v}^{\mathrm{HF}},
$$

and is non-positive because of the variational principle.

DFT replaces the central role of the one-body potential with the ground-state density $n(\mathbf{r})$. From the Hohenberg-Kohn theorem, 23] there is (at most) one $v(\mathbf{r})$ which has a given density as its ground state (for simplicity, we treat only nondegenerate ground state here). From the variational principle introduced in the Hohenberg-Kohn theorem, the ground state energy of system of $N$ electrons and external potential $v(\mathbf{r})$ is 24, 25]

$$
E_{v}=\min _{n \rightarrow N}\left(F[n]+\int v(\mathbf{r}) n(\mathbf{r}) d \mathbf{r}\right),
$$

where $F[n]$ is the universal part of the Hohenberg-Kohn functional, defined as

$$
F[n]=\min _{\Psi \rightarrow n}\left\langle\Psi\left|\hat{T}+\hat{V}_{\mathrm{ee}}\right| \Psi\right\rangle,
$$

where $\hat{T}$ is the kinetic energy operator, $\hat{V}_{\text {ee }}$ is the electron repulsion operator, and the minimization is over all antisymmetric wavefunctions that integrate to density $n(\mathbf{r})$. Denote the minimizer by $\Psi[n]$. The further ansatz of the $\mathrm{KS}$ scheme is that there exists a local multiplicative potential, $v_{\mathrm{S}}(\mathbf{r})$, whose ground-state density for non-interacting fermions matches the interacting one. The total energy in terms of $\mathrm{KS}$ quantities is then

$$
E_{v}=\min _{n}\left(T_{\mathrm{S}}[n]+\int v(\mathbf{r}) n(\mathbf{r}) d \mathbf{r}+E_{\mathrm{H}}[n]+E_{\mathrm{XC}}[n]\right),
$$

where $T_{\mathrm{S}}$ is the kinetic energy of the $\mathrm{KS}$ electrons, $E_{\mathrm{H}}[n]$ is the Hartree energy, and $E_{\mathrm{XC}}[n]$ is the XC energy. The KS wavefunction is $\Phi_{\mathrm{S}}[n]$, which we take here to be a single Slater determinant, as is typical.

We highlight some subtle points that will be important in what follows. The quantum-mechanical operators are known, so each energy components has an obvious functional dependence on the wavefunction, such as

$$
T[\Psi]=-\frac{1}{2} \sum_{i=1}^{N}\left\langle\Psi\left|\nabla_{i}^{2}\right| \Psi\right\rangle
$$

in atomic units. For any Slater determinant $\Phi=\{\chi\}_{N}$ of $N$ orbitals $\chi_{i}(\mathbf{x})$ of space-spin coordinate $\mathbf{x}=(\mathbf{r}, \sigma)$ and $\int d \mathbf{x}=\sum_{\sigma} \int d \mathbf{r}$,

$$
T[\Phi]=\frac{1}{2} \sum_{i=1}^{N} \int d \mathbf{x}\left|\nabla \chi_{i}(\mathbf{x})\right|^{2} .
$$

Density functionals are then defined via the minimizing wavefunctions. The KS kinetic energy is found by setting $\hat{V}_{\text {ee }}=0$ in Eq. 5

$$
T_{\mathrm{S}}[n]=\min _{\Psi \rightarrow n}\langle\Psi|\hat{T}| \Psi\rangle=T\left[\Phi_{\mathrm{S}}[n]\right],
$$

where $\Phi_{\mathrm{S}}[n]$ is minimizer. The exact kinetic energy is

$$
T[n]=T[\Psi[n]] .
$$

These two differ by the correlation kinetic energy:

$$
T_{\mathrm{C}}[n]=T[n]-T_{\mathrm{S}}[n],
$$

which must be non-negative, as $T_{\mathrm{S}}$ is the minimizer of $\hat{T}$ for the given density. Analogously, the exchange energy of $N$ orbitals is:

$$
E_{\mathbf{X}}[\Phi]=-\frac{1}{2} \sum_{i, j}^{N} \iint d \mathbf{x} d \mathbf{x}^{\prime} \frac{\chi_{i}^{*}(\mathbf{x}) \chi_{j}^{*}\left(\mathbf{x}^{\prime}\right) \chi_{j}(\mathbf{x}) \chi_{i}\left(\mathbf{x}^{\prime}\right)}{\left|\mathbf{r}-\mathbf{r}^{\prime}\right|},
$$

which yields the exact exchange density functional in DFT:

$$
E_{\mathrm{X}}[n]=E_{\mathrm{X}}\left[\Phi_{\mathrm{S}}[n]\right] .
$$

The DFT definition of the correlation energy is then

$$
\begin{aligned}
E_{\mathrm{C}}[n] & =\langle\Psi[n]|\hat{H}| \Psi[n]\rangle-\left\langle\Phi_{\mathrm{S}}[n]|\hat{H}| \Phi_{\mathrm{S}}[n]\right\rangle \\
& =T_{\mathrm{C}}[n]+U_{\mathrm{C}}[n],
\end{aligned}
$$

where the potential contribution to correlation is

$$
U_{\mathrm{C}}[n]=V_{\mathrm{ee}}[n]-E_{\mathrm{H}}[n]-E_{\mathrm{X}}[n] .
$$

For weakly correlated systems, such as atoms or most molecules, $T_{\mathrm{C}}$ is only slightly less than $\left|E_{\mathrm{C}}\right|$, so that $E_{\mathrm{C}}+T_{\mathrm{C}}$ which is non-positive, is much smaller in magnitude than either. 
For example, for the He atom, $E_{\mathrm{C}}$ is $-42 \mathrm{mH}, T_{\mathrm{C}}$ is $36 \mathrm{mH}$, and their sum is $-6 \mathrm{mH}$. 26]

There are subtle differences between DFT and wavefunction theory. 27. Since the HF Slater determinant minimizes $\hat{H}$ over all Slater determinants whereas the KS Slater determinant is restricted to orbitals coming from a single multiplicative potential, the definition of correlation energy differs:

$$
\Delta E_{\mathrm{C}, v}=E_{\mathrm{C}, v}^{\mathrm{trad}}-E_{\mathrm{C}}\left[n_{v}\right],
$$

so that

$$
\Delta E_{\mathrm{C}, v}=\left\langle\Phi_{\mathrm{S}}\left[n_{v}\right]|\hat{H}| \Phi_{\mathrm{S}}\left[n_{v}\right]\right\rangle-\left\langle\Phi_{v}^{\mathrm{HF}}|\hat{H}| \Phi_{v}^{\mathrm{HF}}\right\rangle
$$

must be non-negative (although only very slightly, $0.1 \mathrm{mH}$ for $\mathrm{He)}$. [27] A larger difference comes from the difference between the KS and HF Slater determinants, even when they refer to the same density. Define

$$
\Delta T_{\mathrm{S}}^{\mathrm{HF}}[n]=T\left[\Phi_{v[n]}^{\mathrm{HF}}\right]-T_{\mathrm{S}}[n] \geq 0,
$$

where $\Phi_{v[n]}^{\mathrm{HF}}$ is the HF Slater determinant of $v[n](\mathbf{r})$, the onebody potential whose exact density is $n(\mathbf{r})$. We call $\Delta T_{\mathrm{S}}^{\mathrm{HF}}[n]$ the excess non-interacting HF kinetic energy. (In principle, this is found by adjusting $v(\mathbf{r})$ until a HF calculation yields $n(\mathbf{r})$ as its density.) This must be non-negative, as the KS kinetic energy is the minimizer, and can be several $\mathrm{mH}$ for mid-size atoms. [28] Moreover, as $E\left[\Phi_{v[n]}^{\mathrm{HF}}\right] \leq E\left[\Phi_{\mathrm{S}}[n]\right]$, and both Hartree and one-body terms cancel,

$$
\Delta E_{\mathrm{X}}^{\mathrm{HF}}[n] \leq-\Delta T_{\mathrm{S}}^{\mathrm{HF}}[n],
$$

where

$$
\Delta E_{\mathrm{X}}^{\mathrm{HF}}[n]=E_{\mathrm{X}}\left[\Phi_{v[n]}^{\mathrm{HF}}\right]-E_{\mathrm{X}}[n] .
$$

In the special case of atoms, the virial theorem guarantees that the total energy is exactly the negative of the kinetic energy for any minimized calculation, either exact, HF, or DFT with some $\mathrm{XC}$ functional. This implies

$$
\Delta E_{\mathrm{X}}^{\mathrm{HF}}[n]=-2 \Delta T_{\mathrm{S}}^{\mathrm{HF}}[n] \quad \text { (atoms) }
$$

exactly.

\section{ii. Kohn-Sham Inversion}

The problem of finding accurate KS potentials for given densities has been studied almost as long as KS-DFT has been used. [12] There are now many algorithms in existence and use. [29, $30,13,31,32,33,34,35,36$, Some use just the density itself (a pure KS inversion) whereas others are focused on the most relevant case, i.e., densities generated by more accurate and controllable wavefunction calculation, in which case more information is available and can be used. [37, 38, 39, 40, 41] Here we use two pure KS inversion schemes, Zhao-MorrisonParr (ZMP) [31] and Wu-Yang (WY). [32] We always assume the target density is pure-state non-interacting $v$-representable.
The solvers typically work by iteration. A guess for the desired KS potential is made, the KS orbitals are generated, the density is calculated, and the guessed potential is updated according to some algorithms. There are several relevant convergence criteria. The first is the choice of basis set for the inversion algorithm (note that this is independent of the basis set used to generate the target density). Second, there are often guiding functions for the guess. Because typical $\mathrm{XC}$ approximations have incorrect behavior of $v_{\mathrm{S}}(\mathbf{r})$ far from nuclei, one often uses the Fermi-Amaldi (FA) potential, [42] which builds in the correct behavior. In the case of the ZMP procedure, there is a penalty function for errors in the density, which is multiplied by a dimensionless parameter $\lambda$. As $\lambda \rightarrow \infty$, the procedure converges to the target density, but it can become unstable for too large values of $\lambda$.

Traditionally, such inversions are performed on accurate densities in order to gain insight into the exact KS quantities. The paradigmatic example is the extraordinary usefulness of the atomic KS potentials produced by Umrigar and co-workers. [15 16] Knowledge of the positions of the exact KS eigenvalues has been invaluable in tests of time-dependent DFT. 43, But the KS orbital energies are extremely sensitive to details of the potential, but ground-state energy differences (e.g., reaction energies) are not. Below we introduce just those quantities that are relevant to DC-DFT as criteria for sufficient convergence of the KS inversion. For a given inversion recipe, $n^{\mathrm{inv}}(\mathbf{r})$ is a functional of the input or target density, $n(\mathbf{r})$, as are all the KS inversion orbitals and eigenvalues. For any energy functional of the density, we define the inversion error as

$$
\Delta A^{\mathrm{inv}}[n]=A\left[n^{\mathrm{inv}}[n]\right]-A[n],
$$

and we want this error to be sufficiently small so as not to obscure the reaction energies we wish to calculate.

The inversion error in a finite basis comes from several sources. First, a density from a multi-determinantial wavefunction, that is, a correlated density, in a given finite atomic basis set typically cannot be exactly expressed as a KS density in that basis, and a more extensive basis set is required (see [44] for details). This problem only occurs when inverting a correlated target density. Although the basis set of the target density and inversion need not be the same in principle, we usually use same basis set because ZMP and WY typically work with density matrices. Second, one cannot provide infinite flexibility to the KS potential in practice. In the case of ZMP, potential flexibility is limited to the size of the atomic orbital basis set. On the other hand, WY introduces a potential basis, which allows one to increase the flexibility of the potential by increasing the size of that basis. However, increased flexibility of the KS potential in WY may produce orbitals that are very close to the HF orbitals of the same density. [19]. This makes the actual computation of Eq. 22 for the KS kinetic energy (i.e., $A=T_{\mathrm{S}}$ ) impossible because when $n^{\text {inv }}[n]$ approaches to $n$, then simultaneously $T_{\mathrm{S}}$ incorrectly approaches to $T\left[\Phi_{v[n]}^{\mathrm{HF}}\right]$. Nevertheless, in the later section, we 
will show that this ambiguity is sufficiently small as to not invalidate our results.

\section{iii. Density Corrected DFT}

DC-DFT claims that, under well-understood conditions, the SC density in an approximate DFT calculation can contribute significantly to the error, and that such error can usually be reduced by use of a more accurate density. The conventional measure for DFT error in energy is

$$
\Delta E=\tilde{E}[\tilde{n}]-E[n],
$$

where $E$ is the exact energy functional (of Eq. 6 in KS-DFT), and tilde denotes an approximation. One can define a functional error that comes from the approximate $\tilde{E}$ only, by

$$
\Delta E_{\mathrm{F}}=\tilde{E}[n]-E[n]=\tilde{E}_{\mathrm{XC}}[n]-E_{\mathrm{XC}}[n],
$$

where the last equality holds in a KS calculation. The rest of the error comes from the $\tilde{n}(\mathbf{r})$ in the given energy functional;

$$
\Delta E_{\mathrm{D}}=\Delta E-\Delta E_{\mathrm{F}}=\tilde{E}[\tilde{n}]-\tilde{E}[n],
$$

and is called the density-driven error. [2 8]

In DC-DFT, in principle, one should apply the approximate functional to the exact density for DFT calculations whose density-driven errors are significant (about $2 \mathrm{kcal} / \mathrm{mol}$ for small molecules. [22]). By eliminating the density-driven error, the energy usually improves significantly.[7] 8, 9, 10, 11] In practice, calculating highly accurate densities, such as from $\operatorname{CCSD}(T)$, is similar to or more expensive than simply running $\operatorname{CCSD}(T)$ to find energies. For molecular calculations, HF-DFT often suffices to yield significantly improved energetics when densitydriven errors are large, [7] 8,9$] 10$ 11] with little or no additional cost relative to the SC-DFT calculation. However, HF-DFT uses the HF orbitals, simply swapping the HF exchange for the approximate DFT XC, evaluated on the HF orbitals. This procedure ignores $\Delta T_{\mathrm{S}}^{\mathrm{HF}}$, the difference between $\mathrm{HF}$ and $\mathrm{KS}$ kinetic energies.

\section{Results AND Discussion}

Our aim is to test the WY and ZMP KS inversion schemes for use in validating the assumptions underlying HF-DFT. Thus inversion errors in energies must be smaller than the densitydriven errors that are (presumably) being eliminated by the HF-DFT procedure. In this section, we perform inversions targeting $\mathrm{KS}, \mathrm{HF}$, and correlated density, and check the accuracy and precision of the inversion. As our prototypical choices, we consider the $\mathrm{NaCl}$ molecule, both at equilibrium $\left(R_{\mathrm{e}}=2.4 \AA\right)$ and when stretched $\left(R_{\mathrm{s}}=4.5 \AA\right)$. At equilibrium, most calculations with standard functionals are normal (DFT error has negligible density-driven contribution), while most are abnormal when stretched. 2, 8, 9] Our default (standard) functional is PBE, and our default (standard) basis set is aug-cc-pVTZ.
We introduce shorthand notations for the potential basis sets (PBS) for WY; $X, C X, u C X$, and ACX, stand for cc-pVXZ, cc-pCVXZ, unc-cc-pCVXZ, and aug-cc-pCVXZ, respectively,

\begin{tabular}{|c|c|c|c|c|c|c|c|}
\hline \multirow{2}{*}{\multicolumn{2}{|c|}{$\begin{array}{c}\text { Functional } \\
\text { Geometry }\end{array}$}} & \multicolumn{2}{|c|}{$\Delta E^{\mathrm{PBE}}$} & \multicolumn{2}{|c|}{$\Delta E_{\mathrm{rwn}}^{\mathrm{PBE}}$} & \multicolumn{2}{|c|}{$\Delta T_{\mathrm{S}, \mathrm{rxn}}$} \\
\hline & & $R_{\mathrm{e}}$ & $R_{\mathrm{s}}$ & $R_{\mathrm{e}}$ & $R_{\mathrm{s}}$ & $R_{\mathrm{e}}$ & $R_{\mathrm{s}}$ \\
\hline guide & $\lambda$ & \multicolumn{6}{|c|}{ ZMP } \\
\hline \multirow{4}{*}{ FA } & 64 & 11.82 & 11.61 & 3.22 & 3.01 & -534 & -524 \\
\hline & 128 & 7.08 & 6.90 & 1.62 & 1.44 & -309 & -300 \\
\hline & 256 & 4.08 & 3.98 & 0.83 & 0.72 & -178 & -171 \\
\hline & 512 & 2.06 & & 0.37 & & -101 & \\
\hline \multirow{4}{*}{ SVWN } & 64 & 0.40 & 0.39 & 0.01 & 0.00 & -4.88 & $\begin{array}{l}-3.96 \\
\end{array}$ \\
\hline & 128 & 0.27 & 0.26 & 0.01 & 0.00 & -4.46 & -3.65 \\
\hline & 256 & 0.17 & 0.16 & 0.01 & 0.00 & -3.68 & -2.70 \\
\hline & 512 & 0.10 & & 0.01 & & -2.67 & \\
\hline \multirow{4}{*}{ BLYP } & 64 & 0.04 & 0.04 & 0.00 & 0.00 & -0.95 & -0.03 \\
\hline & 128 & 0.02 & 0.02 & 0.00 & 0.00 & -1.28 & -0.22 \\
\hline & 256 & 0.01 & 0.01 & 0.00 & 0.00 & -1.15 & -0.17 \\
\hline & 512 & 0.01 & & 0.00 & & -0.83 & \\
\hline guide & $\overline{\mathrm{PBS}}$ & \multicolumn{6}{|c|}{ WY } \\
\hline \multirow{4}{*}{ FA } & D & 1.81 & 1.82 & -0.44 & -0.43 & 3.08 & 10.12 \\
\hline & $\mathrm{T}$ & 0.53 & 0.34 & 0.06 & -0.13 & -3.01 & 0.40 \\
\hline & $\mathrm{CT}$ & 0.03 & 0.02 & 0.01 & 0.00 & 0.28 & 0.25 \\
\hline & $\mathrm{CQ}$ & 0.00 & 0.00 & 0.00 & 0.00 & 0.10 & 0.16 \\
\hline \multirow{4}{*}{ SVWN } & D & 0.34 & 0.49 & -0.03 & 0.11 & -3.60 & -0.48 \\
\hline & $\mathrm{T}$ & 0.12 & 0.09 & 0.03 & 0.00 & -3.18 & -0.89 \\
\hline & $\mathrm{CT}$ & 0.01 & 0.00 & 0.00 & 0.00 & 0.09 & 0.25 \\
\hline & $\mathrm{CQ}$ & 0.00 & 0.00 & 0.00 & 0.00 & -0.03 & 0.21 \\
\hline \multirow{4}{*}{ BLYP } & D & 0.06 & 0.12 & -0.02 & 0.04 & -0.60 & 1.04 \\
\hline & $\mathrm{T}$ & 0.02 & 0.02 & 0.00 & 0.00 & -1.31 & -0.25 \\
\hline & $\mathrm{CT}$ & 0.00 & 0.00 & 0.00 & 0.00 & 0.36 & 0.26 \\
\hline & CQ & 0.00 & 0.00 & 0.00 & 0.00 & 0.04 & 0.22 \\
\hline
\end{tabular}
where $X(=D, T, Q, 5)$ is the cardinal number of the PBS.

\section{i. Approximate $\mathrm{KS}$ target density}

Table 1: Inversion errors on a KS density for total molecular energy $(\Delta E)$ reaction energy $\left(E_{\mathrm{rxn}}=E(\mathrm{~mol})-E\right.$ (atoms)), and $K S$ kinetic energy for reaction, for $\mathrm{PBE}$ calculations on $\mathrm{NaCl}$, in aug-cc-pVTZ basis. Here, $R_{\mathrm{e}}=2.4 \AA, R_{\mathrm{s}}=4.5 \AA$ and blank cells denote inversions are not converged. All energies are in $\mathrm{mH}$.

A simple consistency check is to take the density from a standard DFT calculation and run inversions to see how accurately we recover the $\mathrm{KS}$ energetic components, for which we have 'exact' answers from the original calculation. To avoid trivial solutions, we used guiding potentials that are not used for SC calculation. In the case of a KS target density, one can easily calculate Eq. 22 for the total energy because $T_{\mathrm{S}}$ is known from the SC-KS calculation. Table 1 shows results for $\mathrm{NaCl}$ with $\mathrm{PBE}$ and its inverted densities. Several important lessons can be drawn from these results. First, errors in this inversion can be driven down to the microhartree range. Second, errors are typically reduced by tightening the convergence parameters, such as larger PBS, larger values of $\lambda$, or using guiding functionals that are close to the original functional that generated the density. Third, when convergence is an issue, total energy converges much faster than energy components, and reaction energies converge much faster than individual 
energies. Fourth, the FA guiding functional converges most slowly here, presumably because the PBE target density was generated from an $X C$ functional yielding a different (and incorrect) asymptotic behavior. Nevertheless, for ZMP/FA, $\lambda=512$ yields sufficiently accurate reaction energies, (subscript rxn hereafter) so we chose to use $\lambda=512$ as our default. In the case of WY, since the accuracy of $\Delta T_{\mathrm{S}, \mathrm{rxn}}$ is greatly improved when we increase PBS from $T$ to $C T$, we chose $C T$, i.e., cc-pCVTZ, as our default potential basis.

\section{ii. Hartree-Fock target density}

\begin{tabular}{cccrrr}
\hline \hline \multicolumn{2}{c}{ Functional } & \multicolumn{2}{c}{$\Delta T_{\mathrm{S}, \text { est }}$} & \multicolumn{2}{c}{$\Delta T_{\mathrm{S}, \mathrm{rxn}, \text { est }}$} \\
\multicolumn{2}{c}{ geometry } & $R_{\mathrm{e}}$ & $R_{\mathrm{s}}$ & $R_{\mathrm{e}}$ & $R_{\mathrm{s}}$ \\
\hline guide & $\lambda$ & \multicolumn{4}{c}{ ZMP } \\
FA & 512 & 2.81 & 2.38 & 0.37 & -0.05 \\
BLYP & 512 & 2.36 & 1.95 & 0.22 & -0.18 \\
\hline guide & PBS & \multicolumn{4}{c}{ WY } \\
\cline { 3 - 6 } FA & $\mathrm{CT}$ & 0.74 & 0.46 & -0.08 & -0.37 \\
& $\mathrm{uC5}$ & 0.26 & 0.24 & 0.18 & 0.16 \\
BLYP & $\mathrm{CT}$ & 0.77 & 0.48 & -0.07 & -0.36 \\
\hline \hline
\end{tabular}

Table 2: Estimated $\Delta T_{\mathrm{S}}^{\mathrm{HF}}$ values (Eq. 30) for equilibrium $\left(R_{\mathrm{e}}=\right.$ $2.4 \AA$ ) and stretched $\left(R_{\mathrm{s}}=4.5 \AA\right.$ ) geometry of $\mathrm{NaCl}$, and for corresponding reaction energies. All energies are in $\mathrm{mH}$.

\begin{tabular}{cccrrr}
\hline \hline \multicolumn{2}{c}{ Functional } & \multicolumn{2}{c}{$\Delta E_{\mathrm{X}, \text { est }}^{\mathrm{HF}}$} & \multicolumn{3}{c}{$\Delta E_{\mathrm{X}, \text { rxn }, \text { est }}^{\mathrm{HF}}$} \\
\multicolumn{2}{c}{ geometry } & $R_{\mathrm{e}}$ & \multicolumn{4}{c}{$R_{\mathrm{s}}$} & $R_{\mathrm{e}}$ & $R_{\mathrm{s}}$ \\
\hline guide & $\lambda$ & \multicolumn{4}{c}{$\mathrm{ZMP}$} \\
FA & 512 & -7.60 & -6.69 & -1.29 & -0.38 \\
BLYP & 512 & -3.70 & -2.94 & -0.70 & 0.06 \\
\hline guide & $\mathrm{PBS}$ & \multicolumn{5}{c}{$\mathrm{WY}$} \\
FA & $\mathrm{CT}$ & -1.70 & -1.13 & 0.30 & 0.88 \\
FA & $\mathrm{uC5}$ & -0.56 & -0.53 & -0.39 & -0.36 \\
BLYP & $\mathrm{CT}$ & -1.68 & -1.04 & 0.14 & 0.78 \\
\hline \hline
\end{tabular}

Table 3: Estimated $\Delta E_{\mathrm{X}}^{\mathrm{HF}}$ values for equilibrium $\left(R_{\mathrm{e}}=2.4 \AA\right)$ and stretched $\left(R_{\mathrm{s}}=4.5 \AA\right)$ geometry of $\mathrm{NaCl}$, and for corresponding reaction energies. All energies are in $\mathrm{mH}$.

Our first non-trivial task is to find $\Delta T_{\mathrm{S}}^{\mathrm{HF}}$, the contribution ignored in a typical HF-DFT calculation. A HF-DFT calculation first runs a HF calculation, then replaces the exchange term with the XC of KS-DFT. For any approximate XC functional, define

$$
\tilde{V}_{\exp }[n]=\int d \mathbf{r} n(\mathbf{r}) v(\mathbf{r})+E_{\mathrm{H}}[n]+\tilde{E}_{\mathrm{XC}}[n],
$$

the contributions to the energy that are known explicitly as functionals of the density. Then,

$$
\tilde{E}^{\mathrm{HF}-\mathrm{DFT}}=T\left[\Phi_{v}^{\mathrm{HF}}\right]+\tilde{V}_{\exp }\left[n_{v}^{\mathrm{HF}}\right] .
$$

However, DFT energies on HF densities, are defined as:

$$
\tilde{E}\left[n^{\mathrm{HF}}\right]=T_{\mathrm{S}}\left[n^{\mathrm{HF}}\right]+\tilde{V}_{\exp }\left[n^{\mathrm{HF}}\right] .
$$

Subtracting Eq. 28 from both sides of Eq. 27 when $n^{\mathrm{HF}}=n_{v}^{\mathrm{HF}}$ yields

$$
\tilde{E}_{v}^{\mathrm{HF}-\mathrm{DFT}}-\tilde{E}\left[n_{v}^{\mathrm{HF}}\right]=T\left[\Phi_{v}^{\mathrm{HF}}\right]-T_{\mathrm{S}}\left[n_{v}^{\mathrm{HF}}\right]=\Delta T_{\mathrm{S}}^{\mathrm{HF}}\left[n_{v}^{\mathrm{HF}}\right] .
$$

However, due to the uncertainty of the inversion, we can not calculate $\tilde{E}\left[n^{\mathrm{HF}}\right]$ nor $T_{\mathrm{S}}\left[n^{\mathrm{HF}}\right]$ exactly. Instead, we calculate $\tilde{E}\left[n^{\mathrm{inv}}\left[n^{\mathrm{HF}}\right]\right]$ or $T_{\mathrm{S}}\left[n^{\mathrm{inv}}\left[n^{\mathrm{HF}}\right]\right]$ via approximate inversion of the HF density. Because the total energy is much less sensitive to small changes of the density (here, inversion error) than individual energy components such as the kinetic energy, for the estimation of $\Delta T_{\mathrm{S}}^{\mathrm{HF}}$, we use

$$
\Delta T_{\mathrm{S}, \mathrm{est}}^{\mathrm{HF}}=\tilde{E}_{v}^{\mathrm{HF}-\mathrm{DFT}}-\tilde{E}\left[n^{\mathrm{inv}}\left[n_{v}^{\mathrm{HF}}\right]\right]
$$

where the subscript est represents the estimated value.

Table 2 reports $\Delta T_{\mathrm{S}, \text { est }}^{\mathrm{HF}}$ values (Eq. 30 for $\mathrm{Na}$ and $\mathrm{Cl}$ atoms and reaction (atomization) energies of $\mathrm{NaCl}$. Even with our standard protocol, the $\Delta T_{\mathrm{S}}^{\mathrm{HF}}$ of ZMP and $\mathrm{WY}$ in molecular energy varies by up to $2 \mathrm{mH}$. But reaction energies are far less sensitive, and here variations are negligible. As the reaction energies of HF-PBE are -148 and $-62 \mathrm{mH}$ for equilibrium and stretched geometry, respectively, these variations are less than $1 \%$ of the reaction energy. Therefore, although obtaining a precise $\Delta T_{\mathrm{S}}^{\mathrm{HF}}$ is not possible with our methodology, estimations can be made precisely enough (within about $\pm 0.5 \mathrm{mH}$ ) for reaction energies to be useful in testing HF-DFT.

In a WY inversion, $\Delta T_{\mathrm{S}, \text { est }}^{\mathrm{HF}}$ becomes very small when PBS is very large, for example, uC5. In the reaction energy calculation of $\mathrm{NaCl}$, this does not cause a severe problem because $\Delta T_{\mathrm{S}}^{\mathrm{HF}}$ itself is very small. However, when $\Delta T_{\mathrm{S}}^{\mathrm{HF}}$ is large, then increasing the size of PBS will eventually cause $\Delta T_{\mathrm{S} \text {,est }}^{\mathrm{HF}}$ to vanish incorrectly. We find that a PBS with the same level of $\zeta$ plus tight core functions is balanced (giving an accurate density but not an unphysically small $\Delta T_{\mathrm{S} \text {,est }}^{\mathrm{HF}}$ ) with an atomic orbital basis. Also, $\Delta T_{\mathrm{S}, \text { est }}^{\mathrm{HF}}$ using different guiding potentials using CT PBS varies less than $0.03 \mathrm{mH}$, indicating that $\mathrm{CT}$ is flexible enough to cover the differences in various guiding potentials. We thus report values of $\Delta T_{\mathrm{S}}^{\mathrm{HF}}$ for reaction energies only, using $\mathrm{WY}$ and assuming uncertainties of $\pm 0.5 \mathrm{mH}$.

To take advantage of error cancellations for $\Delta E_{\mathrm{X}}^{\mathrm{HF}}$, we define

$\Delta E_{\mathrm{X}, \text { est }}^{\mathrm{HF}}=E_{v}^{\mathrm{HF}}-\left\langle\Phi_{\mathrm{S}}\left[n^{\mathrm{inv}}\left[n_{v}^{\mathrm{HF}}\right]\right]|\hat{H}| \Phi_{\mathrm{S}}\left[n^{\mathrm{inv}}\left[n_{v}^{\mathrm{HF}}\right]\right]\right\rangle-\Delta T_{\mathrm{S}, \text { est }}^{\mathrm{HF}}$,

which are shown in Table 3. For each inversion, $\Delta E_{\mathrm{X} \text {,est }}^{\mathrm{HF}}<0$ for total energies. (Eqs. 18 and 19) Also, by compairing Tables 2 and 3 typically $\Delta E_{\mathrm{X} \text {,est }}^{\mathrm{HF}} \approx-2 \Delta T_{\mathrm{S} \text {,est }}^{\mathrm{HF}}$, both for total and reaction energies, as expected from Eq. 21. Deviation from atomic results should be small because $\mathrm{NaCl}$ is a weakly correlated system. In the case of $\mathrm{ZMP}, \Delta E_{\mathrm{X}, \text { est }}^{\mathrm{HF}} \approx-2 \Delta T_{\mathrm{S} \text {,est }}^{\mathrm{HF}}$ is less clear, which shows the limits of the inversion accuracies on this scale of energies. 


\begin{tabular}{|c|c|c|c|c|c|}
\hline \multirow{2}{*}{\multicolumn{2}{|c|}{$\begin{array}{l}\text { Functional } \\
\text { geometry }\end{array}$}} & \multicolumn{2}{|c|}{ 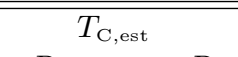 } & \multicolumn{2}{|c|}{$\overline{T_{\mathrm{C}, \mathrm{rxn}, \mathrm{est}}}$} \\
\hline & & $R_{\mathrm{e}}$ & $R_{\mathrm{s}}$ & $R_{\mathrm{e}}$ & $R_{\mathrm{s}}$ \\
\hline guide & $\lambda$ & \multicolumn{4}{|c|}{ ZMP } \\
\hline FA & 512 & 193.10 & 189.01 & 21.77 & 17.68 \\
\hline BLYP & 512 & 193.12 & 189.07 & 21.80 & 17.74 \\
\hline guide & PBS & \multicolumn{4}{|c|}{ WY } \\
\hline FA & CT & 191.94 & 187.77 & 21.85 & 17.68 \\
\hline BLYP & $\mathrm{CT}$ & 191.97 & 187.78 & 21.87 & 17.68 \\
\hline
\end{tabular}

Table 4: Estimated $T_{\mathrm{C}}$ values for equilibrium $\left(R_{\mathrm{e}}=2.4 \AA\right)$ and stretched $\left(R_{\mathrm{s}}=4.5 \AA\right) \mathrm{NaCl}$, and for corresponding reaction energies. Estimations were made using Eq. 33. All energies are in $m H$.

\section{iii. Correlated target density}

Now we consider the inversion when targeting an electron density from a correlated CCSD wavefunction $\left(n^{\mathrm{CC}}\right)$. To check the quality of such an inversion, we extract $T_{\mathrm{C}}$, the kinetic correlation energy, as accurately as practical. Just as for HF, to take advantage of error cancellations, we define, analogous to Eq. 27.

$$
\tilde{E}_{v}^{\mathrm{TC}-\mathrm{DFT}}=T\left[\Psi_{v}^{\mathrm{CC}}\right]+\tilde{V}_{\exp }\left[n_{v}^{\mathrm{CC}}\right]
$$

where $T$ is the value from the CCSD calculation. We can then estimate $T_{\mathrm{C}}$ analogously to Eq. 30

$$
T_{\mathrm{C}, \mathrm{est}}=\tilde{E}_{v}^{\mathrm{TC}-\mathrm{DFT}}-\tilde{E}\left[n^{\mathrm{inv}}\left[n_{v}^{\mathrm{CC}}\right]\right] .
$$

Similar to Table 2 standard ZMP and WY give different estimates of $T_{\mathrm{C}}$ (by approximately $1.2 \mathrm{mH}$ ), but this variation is much smaller in reaction energies (approximately $0.1 \mathrm{mH}$ ).

From Tables 2 and 4 it seems not possible to obtain exact $\Delta T_{\mathrm{S}}^{\mathrm{HF}}$ or $T_{\mathrm{C}}$ values due to the fundamental limitations of the inversion methods. However, the two different inversion algorithms, ZMP and $\mathrm{WY}$, yield consistent estimates for $\Delta T_{\mathrm{S}, \mathrm{rxn}}^{\mathrm{HF}}$ and $T_{\mathrm{C}, \mathrm{rxn}}$. Therefore, we expect inversion can provide $\tilde{E}[n]$ with minor uncertainty, allowing density-driven error estimates with a useful level of accuracy. Since the deviation of the $\Delta T_{\mathrm{S}, \mathrm{rxn} \text {,est }}^{\mathrm{HF}}$ in Table 2 was up to $0.5 \mathrm{mH}$, we expect that the exact inversion results to be within $\pm 0.5 \mathrm{kcal} / \mathrm{mol}$ when calculated under standard inversion conditions. (Note that $1 \mathrm{mH}<$ $1 \mathrm{kcal} / \mathrm{mol}$.) In the next subsection, as a practical application of the inversion, we will present the entire dissociation curves of $\mathrm{NaCl}$ and $\mathrm{OH} \cdot \mathrm{Cl}^{-}$.

\section{iv. Testing HF-DFT}

Previously, it has been argued that the poor description of SC-DFT calculations for dissociation limits of hetero-diatomic molecules was due to density-driven error, and HF-DFT reduces that error. [2, 9] Here we test the argument by quantitatively decomposing the DFT (here, PBE) error using a highly accurate CCSD density as a benchmark. $(\operatorname{CCSD}(T)$ density shows no meaningful differences, see Table S10 in supporting information.) In these calculations, all inversions were performed using the WY algorithm, FA guiding potential, and $\mathrm{CT}$ potential basis (WY/FA/CT). Figure 1 presents dissociation curves of $\mathrm{NaCl}$ molecule.

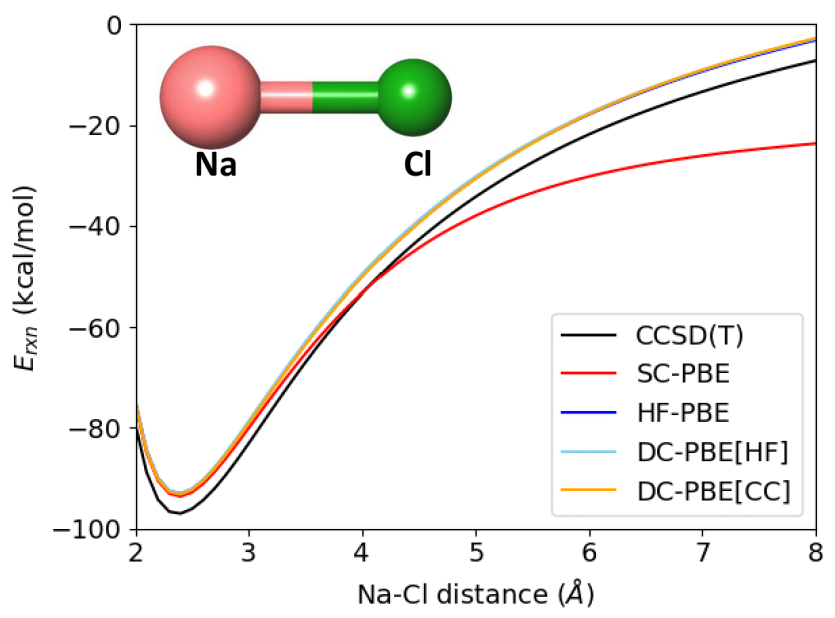

Figure 1: Dissociation curve of $\mathrm{NaCl}$ molecule using $\operatorname{CCSD}(\mathrm{T})$, $S C-P B E, H F-P B E$, and DC-PBE using HF or CCSD density, using $W Y / F A / C T$ inversion. $H F-P B E$ and DC-PBE[HF and $C C]$ are indistinguishable on this scale. The criterion for the inversion imprecision, $\pm 0.5 \mathrm{kcal} / \mathrm{mol}$, is similar to the scale of the thickness of the lines.

While SC-PBE does not account for the correct dissociation behavior, making a huge well-known error in the dissociation limit, [45] DC-PBE with the inversion of the HF or CCSD densities (DC-PBE[HF] and DC-PBE[CC], respectively) correctly capture the dissociation limit, although they are slightly above $\operatorname{CCSD}(\mathrm{T})$. Most importantly, HF-DFT and either of these curves are indistinguishable, showing that HF-DFT differs negligibly from PBE energies evaluated on (essentially) exact densities. This validates the use of HF-DFT as a practical approximation to DC-DFT. Although we have defined density-driven error only for SC-DFT calculations, the densitydriven error can be defined for any approximate $\mathrm{XC}$ energy for non-SC-DFT by replacing $\tilde{n}$ in Eq. 25 to any non-SC density. [5] By doing so, we can calculate the density-driven error of $n^{\mathrm{HF}}$.

To further study the differences between these curves, Figure 2 shows the small differences between curves in Figure 1 . We write

$$
\Delta E_{\mathrm{F}, v}=\Delta E_{v}^{\mathrm{HF}-\mathrm{DFT}}-\Delta E_{\mathrm{D}}\left[n_{v}^{\mathrm{HF}}\right]-\Delta T_{\mathrm{S}}^{\mathrm{HF}}\left[n_{v}^{\mathrm{HF}}\right]
$$

by combining Eqs. 24, 25 and 29 where $\Delta E_{\mathrm{D}}\left[n^{\mathrm{HF}}\right]=$ $\tilde{E}\left[n^{\mathrm{HF}}\right]-\tilde{E}[n]$ is the $\mathrm{PBE}$ density-driven error of the $\mathrm{HF}$ density. Curves are drawn with $\pm 0.5 \mathrm{kcal} / \mathrm{mol}$ bands to represent the uncertainty of the inversion. $\Delta E_{\mathrm{F}}$ in Figure 2 is almost constant regardless of the geometry. Thus, on the scale of the PBE density-driven errors, our imperfect inversions definitely show that the functional error estimated by HF-DFT barely differs from the true value. 


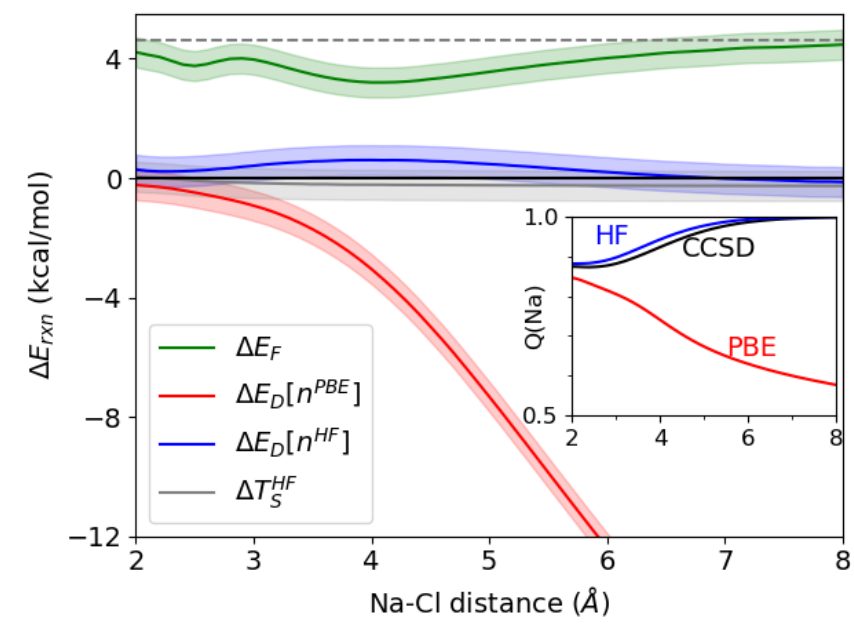

Figure 2: Functional error $\Delta E_{\mathrm{F}}$ and density-driven error $\Delta E_{\mathrm{D}}$ of $n^{\mathrm{PBE}}$ and $n^{\mathrm{HF}}$, and $\Delta T_{\mathrm{S}}^{\mathrm{HF}}$ of $\mathrm{NaCl}$ dissociation curve. Curves are drawn with $\pm 0.5 \mathrm{kcal} / \mathrm{mol}$ bands to represent the uncertainty of the inversion. $\Delta T_{\mathrm{S}}^{\mathrm{HF}}$ is almost indistinguishable from zero. Gray dashed horizontal line $(4.6 \mathrm{kcal} / \mathrm{mol})$ represents $\Delta E_{\mathrm{F}}$ of the reaction $\mathrm{Na}+\mathrm{Cl} \rightarrow \mathrm{Na}^{+}+\mathrm{Cl}^{-}$. Inset shows the IAO population of the $\mathrm{Na}$ atom.

On the other hand, $\Delta E_{\mathrm{D}}\left[n^{\mathrm{PBE}}\right]$ grows strongly with Na$\mathrm{Cl}$ distance, directly showing density delocalization error of PBE. We observed almost zero $\Delta E_{\mathrm{D}}\left[n^{\mathrm{HF}}\right]$ for any geometry in Figure 2 The behavior of $\Delta E_{\mathrm{D}}$ can also be understood from population analysis. Here, we used Mulliken population analysis using intrinsic atomic orbitals (IAO) [46] constructed from KS orbitals of either PBE or an inversion (HF or CCSD). Note that IAO cannot be constructed directly from a correlated wavefunction, which requires $\mathrm{KS}$ inversion. The results are shown in the inset of Figure 2. At $\mathrm{Na}-\mathrm{Cl}$ distance $2 \AA$, the population difference between CCSD and PBE is 0.03 , which has almost no effect on $\Delta E_{\mathrm{D}}\left[n^{\mathrm{PBE}}\right]$. The population difference between $\mathrm{HF}$ and CCSD is maximum near $\mathrm{Na}-\mathrm{Cl}$ distance is $4 \AA$. This difference is reflected in the error curve, where $\Delta E_{\mathrm{D}}\left[n^{\mathrm{HF}}\right]$ becomes slightly positive at that geometry. $\Delta T_{\mathrm{S}}^{\mathrm{HF}}$ is negligibly small everywhere $(-0.08$ and $-0.37 \mathrm{mH}$ for $\mathrm{Na}-\mathrm{Cl}$ distance 2.4 and $4.5 \AA$, see Table 2). The population of $\mathrm{Na}$ atom drops to zero after $8 \AA$, where the triplet state becomes ground state (not shown). [9]

Finally, we point out that the functional error in the reaction $\mathrm{Na}+\mathrm{Cl} \rightarrow \mathrm{Na}^{+}+\mathrm{Cl}^{-}$(marked by the dashed horizontal line in Figure 2) dominates the $\Delta E_{\mathrm{F}}$ in Figure 2. Thus, when measured relative to this limit, the error in a PBE curve evaluated on CC densities, is $<2 \mathrm{kcal} / \mathrm{mol}$ everywhere!

As another example from a previous successful application of HF-DFT, [9] we also analyzed the potential energy curve of the linear $\mathrm{HO} \cdot \mathrm{Cl}^{-}$complex (O-H distance fixed to $1 \AA$ ) in Figure 3 Here, we used augmented PBS (ACT) for WY, due to the $\mathrm{WY}$ convergence issue for $\mathrm{H}-\mathrm{Cl}$ beyond $4 \AA$. SC-PBE shows a significant deviation from $\operatorname{CCSD}(T)$, not only in the

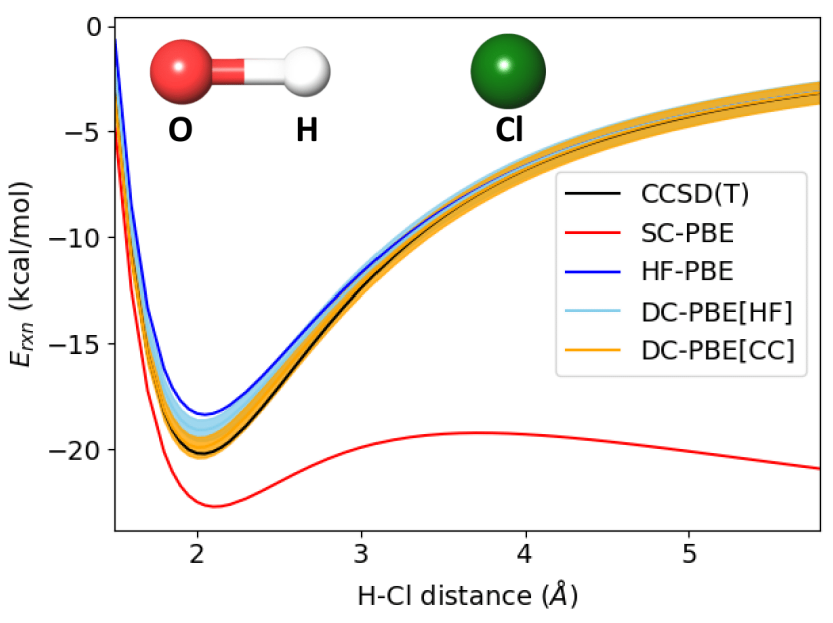

Figure 3: Dissociation curve of $\mathrm{OH} \cdot \mathrm{Cl}^{-}$complex using $C C S D(T), S C-P B E$, HF-PBE, and DC-PBE with $H F$ and $C C S D$ density, using inversion. DC-PBE curves are drawn with $\pm 0.5 \mathrm{kcal} / \mathrm{mol}$ bands to represent the uncertainty of the inversion. All inversions were performed with $W Y / F A / A C T$.

stretched geometry but even in the equilibrium geometry. DCPBE $[C C]$ curve almost coincides with the reference $\operatorname{CCSD}(T)$, showing that $\triangle E_{\mathrm{F}}$ of $\mathrm{PBE}$ functional is almost zero. On the other hand, DC-PBE[HF] and HF-PBE lie slightly higher than $\operatorname{CCSD}(T)$ and differ from each other. HF-PBE, DC-PBE[HF], and DC-PBE $[C C]$ become closer to $\operatorname{CCSD}(\mathrm{T})$ as the $\mathrm{H}-\mathrm{Cl}$ distance increases. So, once again, HF-PBE greatly improves over SC-PBE, but more accurate densities with inversions yield slightly better results.

It is noticeable that the partial charges of the $\mathrm{Cl}$ fragment for any geometries are similar to the charge at the dissociation limit, as in the inset of Figure 4 The behavior of $\Delta E_{\mathrm{D}}\left[n^{\mathrm{PBE}}\right]$ and $\Delta E_{\mathrm{D}}\left[n^{\mathrm{HF}}\right]$ are similar to that of $\mathrm{NaCl}$. However, $\Delta T_{\mathrm{S}}^{\mathrm{HF}}$ is not negligibly small for short $\mathrm{H}-\mathrm{Cl}$ distances; it almost overlaps with $\Delta E_{\mathrm{D}}\left[n^{\mathrm{HF}}\right]$ in Figure $4 \Delta T_{\mathrm{S}}^{\mathrm{HF}}$ needs not be zero for any reaction. The success of $\mathrm{HF}-\mathrm{PBE}$ requires only that it be much smaller than $\left|\triangle E_{\mathrm{D}}\right|$ whenever $\left|\triangle E_{\mathrm{D}}(P B E)\right|>2 \mathrm{kcal} / \mathrm{mol}$.

Of course, in most DFT calculations, the error of SC-DFT does not originate from large $\Delta E_{\mathrm{D}}$. The examples shown here have small $\Delta E_{\mathrm{F}}$, small $\Delta E_{\mathrm{D}}\left[n^{\mathrm{HF}}\right]$, and large $\Delta E_{\mathrm{D}}\left[n^{\mathrm{PBE}}\right]$, and so are greatly improved by the use of HF-DFT.

\section{CONCLUSION}

We have shown here the reliability of present KS inversion methods for the calculation of the density-driven and functional errors of common KS-DFT. Some known issues prohibit exact $\mathrm{KS}$ inversions in localized basis sets. From $\mathrm{KS}$ inversion methods, ZMP and WY, we show that these issues have a minor effect on reaction energies (sub-kcal $/ \mathrm{mol}$ ), when the inversion is performed with proper conditions; such as an ap- 


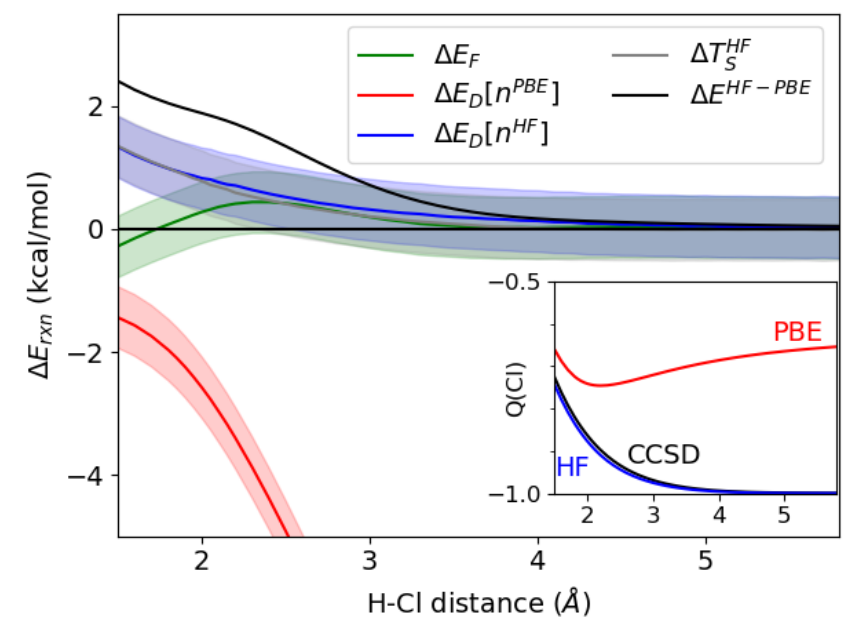

Figure 4: Error components of $\mathrm{PBE}$ and $\Delta T_{\mathrm{S}}^{\mathrm{HF}}$ of $\mathrm{OH} \cdot \mathrm{Cl}^{-}$ dissociation curve. To represent the uncertainty of the inversion, curves are drawn as $\pm 0.5 \mathrm{kcal} / \mathrm{mol}$ bands. $\Delta E_{\mathrm{D}}\left[n^{\mathrm{HF}}\right]$ and $\Delta T_{\mathrm{S}}^{\mathrm{HF}}$ are almost overlapped with each other. Inset shows the IAO population of the Cl atom. The error of HF-PBE is shown in a black solid line with no band because there is no inaccuracy due to inversion.

proximate guiding potential, $\lambda$ in ZMP, or potential basis set in WY. Our recommendations are;

1) In the case of ZMP, a KS-DFT guiding potential works better than FA, even for the inversion of a non-KS density. On the other hand, results are not sensitive to the guiding potential in WY.

2) Large $\lambda$ for ZMP: Practically 512 suffices, larger $\lambda$ may lead to convergence issues.

3) For the potential basis set in WY, using a basis with the same level of $\zeta$ as the atomic orbital basis in addition to tightcore functions.

Under these conditions, one can accurately estimate the density-driven and functional error of common KS-DFT calculations and also estimate the small errors introduced by the HF-DFT procedure. We expect that calculation of these errors will help the development of new $X C$ functionals that reduce both $\Delta E_{\mathrm{D}}$ and $\Delta E_{\mathrm{F}}$.

\section{Computational Details}

Coupled cluster singles, doubles (CCSD) is used as a reference density, while perturbative triples $(\operatorname{CCSD}(T))$ is used as a reference of energy. All HF, DFT, and CC calculations were performed using PySCF program package. [47] Since we used PBE[48] as the default energy functional, FA, SVWN, 49, 50] and BLYP [51, 52] are tested as guiding potentials for inversions. The unrestricted scheme is used for open-shell systems. No frozen-core approximations were made for CC calculations. The aug-cc-pVTZ atomic orbital basis set is used for both $\mathrm{NaCl}$ molecule, and $\mathrm{HO} \cdot \mathrm{Cl}^{-}$complex.[53 54 55] We set gradi- ent converge threshold (conv_tol_grad attribute in SCF base class in PySCF) for $\mathrm{HF}$ to $1 \mathrm{e}-7$, to generate accurate reference $\mathrm{HF}$ determinant for $\mathrm{CC}$ calculations. For the convergence of PBE on the stretched molecules, we set level_shift $=0.2$ and conv_check=False. All ZMP and WY calculations were conducted with our codes. For ZMP, we used direct inversion of iterative subspace algorithm[56] to accelerate convergence. We solved ZMP equations self-consistently for a $\lambda$ and used the output density matrix as an initial guess of the next ZMP equation with a larger $\lambda$. We say ZMP fails to converge at $\lambda^{\prime}$ when it fails to converge when the initial guess density matrix is from $\lambda^{\prime}-1$. For $W Y$, we used the BroydenâĂȘFletcherâĂSGoldfarbâĂȘShanno algorithm [57] implemented in SciPy [58] for the optimization of the KS potential.

\section{Associated Content}

Tables for all inversion results corresponding to all tables and figures are provided. Also, we present inversion results of CCSD and $\operatorname{CCSD}(\mathrm{T})$ densities for $\mathrm{NaCl}$, and ZMP results for $\mathrm{HO} \cdot \mathrm{Cl}^{-}$complex. Please see supporting information.

\section{ACKNOWLEDGEMENT}

This work at Yonsei University was supported by the grant from the Korean Research Foundation (2020R1A2C2007468). KB acknowledges NSF for Grant CHE 1856165.

\section{REFERENCES}

[1] Walter Kohn and Lu Jeu Sham. Self-consistent equations including exchange and correlation effects. Physical review, 140(4A):A1133, 1965.

[2] Min-Cheol Kim, Eunji Sim, and Kieron Burke. Understanding and reducing errors in density functional calculations. Physical review letters, 111(7):073003, 2013.

[3] Aron J Cohen, Paula Mori-Sánchez, and Weitao Yang. Insights into current limitations of density functional theory. Science, 321(5890):792-794, 2008.

[4] Adam Wasserman, Jonathan Nafziger, Kaili Jiang, MinCheol Kim, Eunji Sim, and Kieron Burke. The importance of being inconsistent. Annual review of physical chemistry, 68:555-581, 2017.

[5] Stefan Vuckovic, Suhwan Song, John Kozlowski, Eunji Sim, and Kieron Burke. Density functional analysis: The theory of density-corrected dft. Journal of chemical theory and computation, 15(12):6636-6646, 2019.

[6] http://tccl.yonsei.ac.kr/mediawiki/index. php/DC-DFT 
[7] Min-Cheol Kim, Eunji Sim, and Kieron Burke. Communication: Avoiding unbound anions in density functional calculations. 134, 2011.

[8] Min-Cheol Kim, Eunji Sim, and Kieron Burke. Ions in solution: Density corrected density functional theory (dc$\mathrm{dft}$ ). The Journal of chemical physics, 140(18):18A528, 2014.

[9] Min-Cheol Kim, Hansol Park, Suyeon Son, Eunji Sim, and Kieron Burke. Improved dft potential energy surfaces via improved densities. The journal of physical chemistry letters, 6(19):3802-3807, 2015.

[10] Suhwan Song, Min-Cheol Kim, Eunji Sim, Anouar Benali, Olle Heinonen, and Kieron Burke. Benchmarks and reliable $\mathrm{dft}$ results for spin gaps of small ligand fe (ii) complexes. Journal of chemical theory and computation, 14(5):2304-2311, 2018.

[11] Yeil Kim, Suhwan Song, Eunji Sim, and Kieron Burke. Halogen and chalcogen binding dominated by densitydriven errors. The journal of physical chemistry letters, 10(2):295-301, 2018.

[12] Carl-Olof Almbladh and Antonio Carlos Pedroza. Densityfunctional exchange-correlation potentials and orbital eigenvalues for light atoms. Physical Review A, 29(5):2322, 1984.

[13] R Van Leeuwen and EJ Baerends. Exchange-correlation potential with correct asymptotic behavior. Physical Review A, 49(4):2421, 1994.

[14] Oleg V Gritsenko and Evert Jan Baerends. Effect of molecular dissociation on the exchange-correlation kohnsham potential. Physical Review A, 54(3):1957, 1996.

[15] Cyrus J Umrigar and Xavier Gonze. Accurate exchangecorrelation potentials and total-energy components for the helium isoelectronic series. Physical Review A, 50(5):3827, 1994.

[16] Dana A Browne, Joseph Callaway, Jerry P Draayer, Richard W Haymaker, Rajiv K Kalia, Joel E Tohline, and Priya Vashishta. Comparison of approximate and exact density functionals: A quantum monte carlo study, umrigar, cyrus $\mathrm{j}$ and gonze, xavier, in high performance computing and its applications in the physical sciences: Proceedings of the mardi gras' 93 conference. In High Performance Computing and its Applications in the Physical Sciences: Proceedings of the Mardi Gras' 93 Conference, pages 1-274. World Scientific, 1994.

[17] Rodney J Bartlett, Ireneusz Grabowski, So Hirata, and Stanislav Ivanov. The exchange-correlation potential in ab initio density functional theory. The Journal of chemical physics, 122(3):034104, 2005.
[18] So Hirata, Stanislav Ivanov, Ireneusz Grabowski, Rodney J Bartlett, Kieron Burke, and James D Talman. Can optimized effective potentials be determined uniquely? The Journal of Chemical Physics, 115(4):1635-1649, 2001.

[19] VN Staroverov, GE Scuseria, and ER Davidson. Optimized effective potentials yielding hartree-fock energies and densities. The Journal of chemical physics, 124(14):141103, 2006.

[20] Tim Heaton-Burgess, Felipe A Bulat, and Weitao Yang. Optimized effective potentials in finite basis sets. Physical review letters, 98(25):256401, 2007.

[21] Christian Kollmar and Frank Neese. The static response function in kohn-sham theory: An appropriate basis for its matrix representation in case of finite ao basis sets. The Journal of chemical physics, 141(13):134106, 2014.

[22] Eunji Sim, Suhwan Song, and Kieron Burke. Quantifying density errors in $\mathrm{dft}$. The Journal of Physical Chemistry Letters, 9(22):6385-6392, 2018.

[23] Pierre Hohenberg and Walter Kohn. Inhomogeneous electron gas. Physical review, 136(3B):B864, 1964.

[24] Mel Levy. Universal variational functionals of electron densities, first-order density matrices, and natural spin-orbitals and solution of the v-representability problem. Proceedings of the National Academy of Sciences, 76(12):6062-6065, 1979.

[25] Elliott Lieb. Density functionals for couiomb systems. International Journal of Quantum Chemistry, 24(3):243277, 1983.

[26] Chien-Jung Huang and CJ Umrigar. Local correlation energies of two-electron atoms and model systems. Physical Review A, 56(1):290, 1997.

[27] Eberhard KU Gross, M Petersilka, and T Grabo. Conventional quantum chemical correlation energy versus density-functional correlation energy. 629:42-53, 1996.

[28] Andreas Görling and Matthias Ernzerhof. Energy differences between kohn-sham and hartree-fock wave functions yielding the same electron density. Physical Review A, 51(6):4501, 1995.

[29] Andreas Görling. Kohn-sham potentials and wave functions from electron densities. Physical Review A, 46(7):3753, 1992.

[30] Yue Wang and Robert G Parr. Construction of exact kohn-sham orbitals from a given electron density. Physical Review A, 47(3):R1591, 1993. 
[31] Qingsheng Zhao, Robert C Morrison, and Robert G Parr. From electron densities to kohn-sham kinetic energies, orbital energies, exchange-correlation potentials, and exchange-correlation energies. Physical Review A, 50(3):2138, 1994.

[32] Qin $W u$ and Weitao Yang. A direct optimization method for calculating density functionals and exchangecorrelation potentials from electron densities. The Journal of chemical physics, 118(6):2498-2509, 2003.

[33] Ilya G Ryabinkin and Viktor N Staroverov. Determination of kohn-sham effective potentials from electron densities using the differential virial theorem. The Journal of chemical physics, 137(16):164113, 2012.

[34] Xing Zhang and Emily A Carter. Kohn-sham potentials from electron densities using a matrix representation within finite atomic orbital basis sets. The Journal of chemical physics, 148(3):034105, 2018.

[35] Kati Finzel, Paul W Ayers, and Patrick Bultinck. A simple algorithm for the kohn-sham inversion problem applicable to general target densities. Theoretical Chemistry Accounts, 137(3):30, 2018.

[36] Bikash Kanungo, Paul M Zimmerman, and Vikram Gavini. Exact exchange-correlation potentials from ground-state electron densities. Nature communications, 10(1):1-9, 2019.

[37] Ilya G Ryabinkin, Sviataslau V Kohut, and Viktor N Staroverov. Reduction of electronic wave functions to kohn-sham effective potentials. Physical review letters, 115(8):083001, 2015.

[38] Rogelio Cuevas-Saavedra, Paul W Ayers, and Viktor N Staroverov. Kohn-sham exchange-correlation potentials from second-order reduced density matrices. The Journal of chemical physics, 143(24):244116, 2015.

[39] Rogelio Cuevas-Saavedra and Viktor N Staroverov. Exact expressions for the kohn-sham exchange-correlation potential in terms of wave-function-based quantities. Molecular Physics, 114(7-8):1050-1058, 2016.

[40] Egor Ospadov, llya G Ryabinkin, and Viktor N Staroverov. Improved method for generating exchange-correlation potentials from electronic wave functions. The Journal of chemical physics, 146(8):084103, 2017.

[41] Egor Ospadov and Viktor N Staroverov. Construction of fermi potentials from electronic wave functions. Journal of chemical theory and computation, 14(8):4246-4253, 2018.

[42] Enrico Fermi and Edoardo Amaldi. Le orbite infinito degli elementi. Accad. Ital. Rome., 6:117, 1934.
[43] A Savin, Cyrus J Umrigar, and Xavier Gonze. Relationship of kohn-sham eigenvalues to excitation energies. Chemical Physics Letters, 288(2-4):391-395, 1998.

[44] Istvan Mayer, Imre Papai, Imre Bako, and Agnes Nagy. Conceptual problem with calculating electron densities in finite basis density functional theory. Journal of chemical theory and computation, 13(9):3961-3963, 2017.

[45] Adrienn Ruzsinszky, John P Perdew, Gábor I Csonka, Oleg A Vydrov, and Gustavo E Scuseria. Spurious fractional charge on dissociated atoms: Pervasive and resilient self-interaction error of common density functionals. The Journal of chemical physics, 125(19):194112, 2006.

[46] Gerald Knizia. Intrinsic atomic orbitals: An unbiased bridge between quantum theory and chemical concepts. Journal of chemical theory and computation, 9(11):48344843, 2013.

[47] Qiming Sun, Timothy C. Berkelbach, Nick S. Blunt, George H. Booth, Sheng Guo, Zhendong Li, Junzi Liu, James D. McClain, Elvira R. Sayfutyarova, Sandeep Sharma, Sebastian Wouters, and Garnet Kin-Lic Chan. Pyscf: the python-based simulations of chemistry framework. Wiley Interdisciplinary Reviews: Computational Molecular Science, 8(1):e1340, 2018.

[48] John P Perdew, Kieron Burke, and Matthias Ernzerhof. Generalized gradient approximation made simple. Physical review letters, 77(18):3865, 1996.

[49] Paul AM Dirac. Note on exchange phenomena in the thomas atom. In Mathematical Proceedings of the Cambridge Philosophical Society, volume 26, pages 376-385. Cambridge University Press, 1930.

[50] Seymour H Vosko, Leslie Wilk, and Marwan Nusair. Accurate spin-dependent electron liquid correlation energies for local spin density calculations: a critical analysis. Canadian Journal of physics, 58(8):1200-1211, 1980.

[51] Axel D Becke. Density-functional exchange-energy approximation with correct asymptotic behavior. Physical review $A$, 38(6):3098, 1988.

[52] Chengteh Lee, Weitao Yang, and Robert G Parr. Development of the colle-salvetti correlation-energy formula into a functional of the electron density. Physical review $B, 37(2): 785,1988$.

[53] Thom H. Dunning. Gaussian basis sets for use in correlated molecular calculations. i. the atoms boron through neon and hydrogen. The Journal of Chemical Physics, 90(2):1007-1023, 1989.

[54] Rick A Kendall, Thom H Dunning Jr, and Robert J Harrison. Electron affinities of the first-row atoms revisited. 
systematic basis sets and wave functions. The Journal of Chemical Physics, 96(9):6796-6806, 1992.

[55] David E Woon and Thom H Dunning Jr. Gaussian basis sets for use in correlated molecular calculations. iii. the atoms aluminum through argon. The Journal of Chemical Physics, 98(2):1358-1371, 1993.

[56] Péter Pulay. Convergence acceleration of iterative sequences. the case of scf iteration. Chemical Physics Letters, 73(2):393-398, 1980.

[57] Jorge Nocedal and Stephen Wright. Numerical optimization. Springer Science \& Business Media, 2006.

[58] Pauli Virtanen, Ralf Gommers, Travis E. Oliphant, Matt Haberland, Tyler Reddy, David Cournapeau, Evgeni Burovski, Pearu Peterson, Warren Weckesser, Jonathan Bright, Stéfan J. van der Walt, Matthew Brett, Joshua Wilson, K. Jarrod Millman, Nikolay Mayorov, Andrew R. J. Nelson, Eric Jones, Robert Kern, Eric Larson, CJ Carey, IIhan Polat, Yu Feng, Eric W. Moore, Jake Vand erPlas, Denis Laxalde, Josef Perktold, Robert Cimrman, lan Henriksen, E. A. Quintero, Charles R Harris, Anne M. Archibald, Antônio H. Ribeiro, Fabian Pedregosa, Paul van Mulbregt, and SciPy 1. 0 Contributors. SciPy 1.0: Fundamental Algorithms for Scientific Computing in Python. Nature Methods, 17:261-272, 2020. 